\title{
The Research of the Safety Level of Nations Special Equipment Based on Analytic Hierarchy Theory
}

\author{
*WeiPing OuYang ${ }^{\mathrm{a}}$, Qi Zhou ${ }^{\mathrm{b}}$, Jun Qiu
}

Shanghai Institute of Special Equipment Inspection and Technical Research, Shanghai China

aouyangweipingvi@163.com

bzhouq@ssei.com

cqiuj@ssei.com

*Weiping Ouyang

\begin{abstract}
Keywords: safety level of special equipment, analytic hierarchy theory, comparison and analysis Abstract: The paper expounds the necessity of scientific and objective evaluating and comparing the safety level of special equipment. Through the introduction of analytic hierarchy theory, the paper establishes a comparative analysis model of the safety level of the special equipment, and establishes the price criterion of the model index, and ensures that the evaluation conclusion is scientific and accurate. In the engineering application, several typical countries and China are selected to compare and analyze the safety level of special equipment, and some problems need to be solved are obtained.
\end{abstract}

\section{preface}

The special equipment is defined as eight categories like boilers, pressure vessels (including gas cylinders), pressure pipes, elevators, lifting machinery, passenger transport, large recreational facilities and field (factory) special motor vehicles for its greater risk and close association to peoples life safety. With the continuous development of society, people pay much more attention to security of the safe operation of special equipment. For this purpose, State Administration of quality supervision and inspection set up the corresponding inspection agencies and regulatory organization to carry out supervision and inspection tasks of the special equipment, in order to ensure the safe operation of the equipment ${ }^{[1]}$. There is no precise evaluation model and theory available now to evaluate the relative safety level of China when compare to other countries, this restricts the development of China's special equipment safety level at some extent. For this purpose, the SSEI built a working group in particular to explore the proper evaluation method and establish a comparative analysis model, several countries, the economic and social development stage is relatively close to China, are selected as the China's comparative analysis object to make clear the development stage of the china's special equipment safety level. The future development direction of China's special equipment safety management was obtained.

\section{analytic hierarchy theory}

Structure methods are popular method employed to conducting system evaluation. Among those methods, the analytic hierarchy theory ${ }^{[2]}$ (AHP method) is suggested by professor Sarti from the University of Pittsburgh, which is the most representative and the most widely used method between structure methods. The AHP method be able to express a complex problem, the relative 
importance of each constitution factor of the complex problem of the process is obtained by the calculation of the specific rules, into an ordered hierarchical structure. The whole solving process of the problem covers the risk source identification and differentiation, the importance of different risk sources and equipment safety performance calculation ${ }^{[3]}$.

The concrete method is to decompose the problem the problem into multiple levels, each level is consisted by several influence factors ${ }^{[4]}$. As Fig.1 shows, a multi - layer structure model of the target that needs to be solved is formed according to the logical relationship between the factors. The top layer in Figure.1 is called the target layer (T level), which means the target to be analyzed; and The bottom layer of the model is named the lowest level (the a level), which means each component factor of this layer is the basic factor; The layer between the $\mathrm{T}$ layer and the a layer is the intermediate layer, which represents the intermediate link of the solving process of the target. After the determining and arranging the hierarchy of all the influencing factors as shown in Figure 1, the relative importance of each factor is scored by the experts, and a comparison judgment matrix was established according to this, the importance of each factors can be obtained through solving the comparison judgment matrix. Evaluation criteria or method of the lowest level's factor need to be established also, the general method presently is: quantitative evaluation, qualitative evaluation method and test evaluation method etc, and the actual state evaluation $\left(\mathrm{I}_{\mathrm{i}}\right)$ of each factor can be obtained accordingly. Therefore, the state of the safety level of special equipment could be expressed by comprehensive evaluation index (D), as equation following shows:

$$
\mathrm{D}=\sum_{1}^{n} \omega_{\mathrm{i}} \times \mathrm{I}_{\mathrm{i}} \quad(\mathrm{i}=1 、 2 、, \ldots, 0 \leq \mathrm{D} \leq 1) \quad \text { Equ. } 1
$$

Among them, $\omega_{i}$ is the importance of each factor. The comprehensive evaluation index (D) is the value after normalized. According to the characteristics of safety level of special equipment, the safety level corresponding to the different range of $\mathrm{D}$ are distinguished and defined by experts as table. 1 shows.

Table.1 the safety level of different range of D

\begin{tabular}{|c|c|}
\hline The value of $\mathrm{D}$ & Safety level \\
\hline$\geq 0.8$ & high \\
\hline $0.6 \leq \mathrm{D}<0.8$ & Relatively high \\
\hline $0.4 \leq \mathrm{D}<0.6$ & normal \\
\hline $\mathrm{D}<0.4$ & low \\
\hline
\end{tabular}




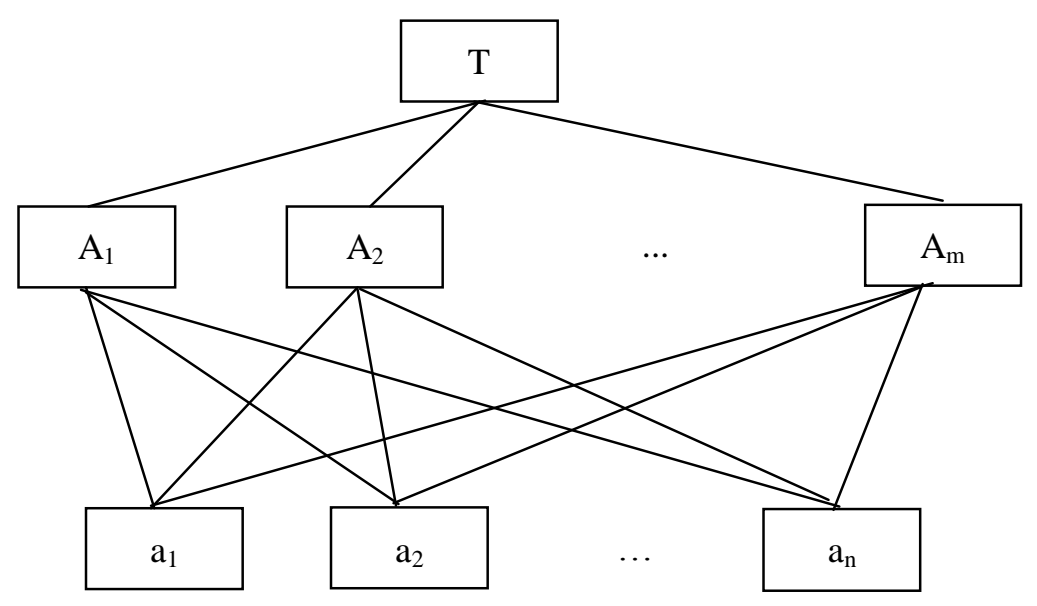

Fig.1 hierarchical structure model

\section{construction of the comparative analysis model}

\section{Target decomposition and hierarchical division}

The target could be divided into five associated levels, and the factor of each level is decomposed from the relative upper level's factor, and the constitution situation of top three level of the safety level will be introduced here for confidential consideration. As shown in Fig.2, the top layer safety level could divided into four different factors as equipment essential safety(EES), group safety awareness(GSA), laws and regulation system(LRS) and accident emergency disposal ability(EDA), and these four factors could be further divided as shown at Fig.2.

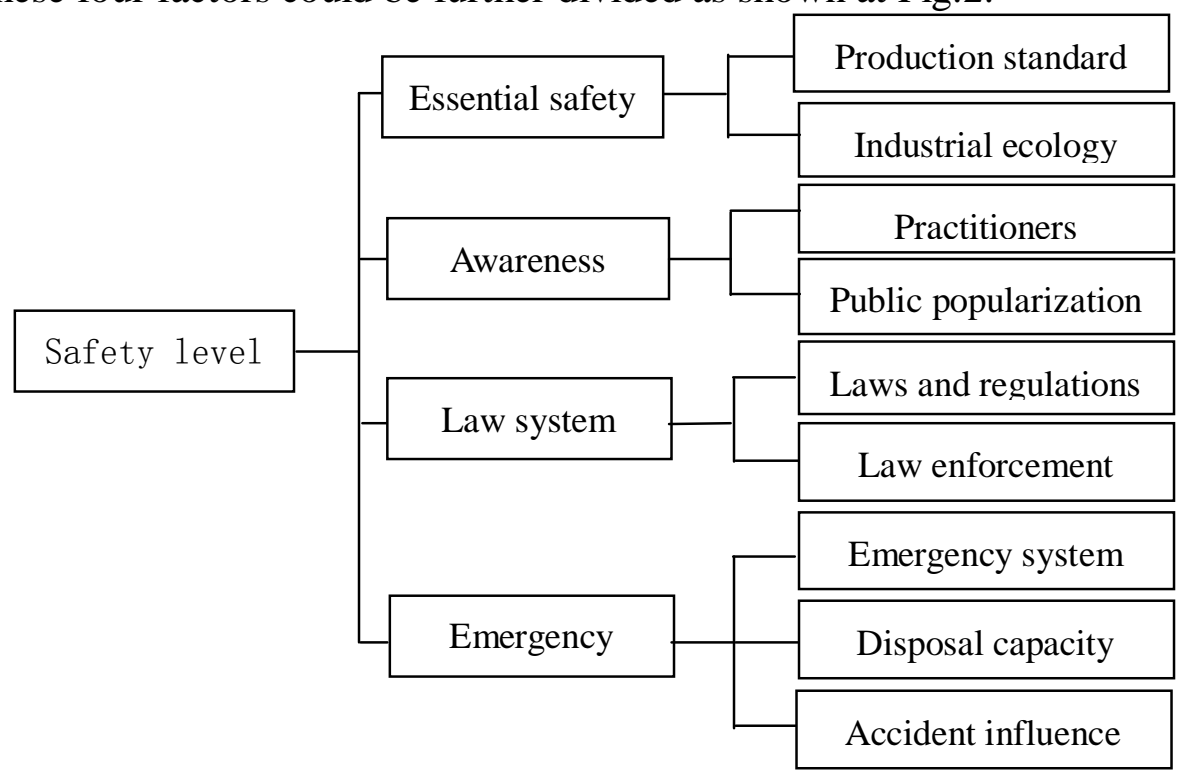

Fig.2 the special equipment safety level sketch map

Determination of the relative importance of the factor of the comparative analysis model and establishing the comparison matrix

After the establishment of a comparative analysis model, the effect of each factor on the upper layer is need to be confirmed, that is, the comparison matrix ought to be obtained. The relative importance between the factors was determined through the experts judgment in accordance with the 1-9quantitative scale (as table2 shows). 
Table. 2 the value and meaning of 1-9 quantitative scale

\begin{tabular}{|c|l|}
\hline scaling & \multicolumn{1}{c|}{ meaning } \\
\hline 1 & Same importance while two factors compare \\
\hline 3 & The former little importance while two factors compare \\
\hline 5 & The former is obviously important while two factors compare \\
\hline 7 & The former is strongly important while two factors compare \\
\hline 9 & The former is extremely important while two factors compare \\
\hline $2,4,6,8$ & The intermediate value of the above comparison \\
\hline
\end{tabular}

The relative importance of influencing factors of the same level (as shown in Fig.2) is evaluated by a professional staff while in accordance with the table 2 and the mathematical statistics was conducted to obtain the symmetric matrix of the intermediate A to target layer $\mathrm{T}$ (like the second layer four factors to the top layer as shown at Fig.2). The maximum eigenvector of the matrix is obtained by using the sum product method, and the maximum eigenvector $\omega=(0.35,0.35$, $0.18,0.12)^{t}$. The comparative matrix and the maximum eigenvector of the third layers factor ai to the second layers factor Ai could be gained similarly.

Table. 3 the comparative matrix of the second layer of the model

\begin{tabular}{|c|c|c|c|c|}
\hline $\mathrm{T}$ & $\mathrm{A} 1$ & $\mathrm{~A} 2$ & $\mathrm{~A} 3$ & $\mathrm{~A} 4$ \\
\hline $\mathrm{A} 1$ & 1 & 1 & 2 & 3 \\
\hline $\mathrm{A} 2$ & 1 & 1 & 2 & 3 \\
\hline A3 & $1 / 2$ & $1 / 2$ & 1 & $3 / 2$ \\
\hline A4 & $1 / 3$ & $1 / 3$ & $2 / 3$ & 1 \\
\hline
\end{tabular}

\section{The consistency verification of the comparative matrix}

Sometimes the relative importance of the factors solved from the comparative matrix is not conform the actual situation and this could be avoid by the verification consistency of the comparison matrix by calculating the maximum characteristic root ( $\lambda \max )$ of each comparative matrix as the equation following shows:

$$
\lambda_{\max }=\sum_{1}^{n} \frac{(A \omega)_{i}}{n \omega_{i}}(i=1,2, \ldots n)
$$

Through calculation, the maximum characteristic root $\lambda \max =3.024$.

And the Consistency Index (CI) of the second layers factor to the target layer of matrix $\mathrm{T}$ could be calculated as following:

$$
\begin{aligned}
& \mathrm{CI}=\frac{\lambda_{\text {max }}-\mathrm{n}}{n-1} \\
& =0.012
\end{aligned}
$$

The random index (RI) of the comparative matrix could be obtained through inquiry the professional table, while $\mathrm{RI}=0.58$, therefore, the Consistency Ratio (CR) could be got: 


$$
\begin{aligned}
& \mathrm{CR}=\frac{\mathrm{CI}}{\mathrm{RI}} \quad \text { (Equ.4) } \\
& =0.02<0.10
\end{aligned}
$$

It could be found in the professional material that when CR value $<0.10$, the consistency of the comparative matrix will be considered acceptable. The consistency verification of other layers comparative matrix could be conducted, similarly, and the construction of the comparative analysis model of the special equipment safety level is proper through calculation.

\section{Engineering application of the comparative analysis model}

\section{Selection of comparative objects}

China's special equipment supervision has been carried out for several decades, especially in recent years, with the combination of the advanced management experience of the West, a obvious improvement of the safety level of our country's special equipment has brought out. Through the research and pre evaluation of SSEI research group, the safety level of China special equipment is similar to that of the medium developed countries, several typical country special equipment management, social and economic level close or slightly higher to our country are selected as comparison object. After the analysis of the research group, Poland, Russia, South Africa, Brazil and Argentina five medium developed countries are selected as China's comparative object for special equipment safety level.

\section{Comparison and analysis of results}

6 working groups for the selected countries were found in SSEI research team responsible for the implementation of the comparative analysis model of the special equipment safety level in detail, and the work carried out were include accessing the related information, laws, regulations and management system of special equipment, acquire the related data from the selected countries official website and the questionnaire investigation of the local people. All the accessed information were sorted and analyzed, and converted into the lowest layer factors actual state evaluation, the D value of this country's special equipment safety level could be calculated as the Eq.4 shows, the D value of the six selected countries are shown at the table 4.

Table. 4 the comprehensive evaluation index of China and the comparison object

\begin{tabular}{|c|c|}
\hline country & D value of safety level \\
\hline Poland & 0.84 \\
\hline China & 0.80 \\
\hline Russia & 0.78 \\
\hline Argentina & 0.76 \\
\hline Brazil & 0.76 \\
\hline South Africa & 0.71 \\
\hline
\end{tabular}

Through the analysis and comparison, China's actual special equipment safety level could be a relatively clear observed including its advantages and disadvantages. By comparative analysis, it can be known that Poland though has no national standards, yet has the CE certification as the same as the other developed countries in the EU, has done better in the laws and regulations, the safety level of D is relatively high therefore; similarly to Poland, Argentina has no national standards too and the current European standards and the United States are both in use, the score was slightly lower for the gap in law enforcement and emergency response capabilities when compare to the Poland; China and Russia are similar in safety level, both of two countries are implementing domestic standards and there are specialized agencies and regulations to manage the special 
equipment of the country, Though there are some differences between the two countries in the emergency treatment and accident rate, but the overall level is similar to each other. As can be seen from table four, the developed countries in Europe and the United States have maintained a high level of safety level of special equipment, and have the relative perfect system. China and Russia, as the representative of the emerging countries, have catching up in the management of special equipment, there is still a gap in the standard formulation and public security education and awareness especially ordinary people in China, this has brought great challenges to the accident emergency disposal work.

\section{Conclusion}

The paper introduced how to use the analytic hierarchy theory to build a comparative analysis model for national special equipment safety level evaluation, through building up the correct model, a few typical countries and China are selected to compared to each other on the national special equipment safety level, and obtained the following conclusions:

(1) Analytic hierarchy theory is applicable to the evaluation of the safety level of national special equipment. The conclusion is detailed and concrete by the way of quantitative, qualitative and questionnaire survey, which is helpful to further strengthen of the supervision work of special equipment.

(2) Through the engineering application of the model, we can know that our country's special equipment safety level is in the middle level during the medium developed countries. This shows that our government is basically in place for the supervision of special equipment. However, the gap in the standard and the awareness of the public safety should be paid enough attention, and gradually improve and strengthen in the future work..

\section{Reference}

[1] AICHE. Dow's Chemical Explosion Index Guide. First Edition.1994

[2] Banon H, Biffs J M, Irvine HM. Seismic damage in reinforced concrete frames. Journal of Structural Engineering,1991,107(9):1713-1729.

[3] Wang ML, Shah S P. Reinforced concrete hysteresis model based on the damage concept Earthquake Engineering and Structural Dynamics,1997, 15(8): 993-1003.

[4] Park Y J, Ang AH-S. Mechanistric seismic damage model for reinforced concrete. Journal of Structural Engineering,1995,111(4):722-739. 